\title{
Open charm production in deep inelastic scattering at next-to-leading order at HERA
}

Brian W. Harris RECEIVEO

HEP Theory Group, Argonne National Laboratory, Argonne, IL 60439, USA

\begin{abstract}
An introduction and overview of charm production in deep inelastic scattering at HERA is given. The existing next-to-leading order perturbative QCD calculations are then reviewed, and key results are summarized. Finally, comparisons are made with the most recent HERA data, and unresolved issues are highlighted.
\end{abstract}

\section{Introduction}

Electromagnetic interactions have long been used to study both hadronic structure and strong interaction dynamics. Examples include deep inelastic leptonnucleon scattering, hadroproduction of lepton pairs, the production of photons with large transverse momenta, and various photoproduction processes involving scattering of real or very low mass virtual photons from hadrons. In particular, heavy quark production in deep inelastic electron-proton scattering (DIS) is calculable in QCD and provides information on the gluonic content of the proton which is complementary to that obtained in direct photon production or structure function scaling violation measurements. In addition, the scale of the hard scattering may be large relative to the mass of the charm quark, thus allowing one to study whether and when to treat the charm as a parton.

Early measurements of open charm production in neutral current DIS, performed by the Berkeley-Fermilab-Princeton (BFP) [1] and European Muon Collaboration (EMC) [2] experiments, touched upon the topics relevant for HERA today: production mechanism, charm fragmentation, gluon parton distribution function extraction, and charm contribution to the proton structure function. See [3] for a review of these experiments. Activity in this area has increased recently with new data available from the $\mathrm{H} 1$ [4] and ZEUS [5] experiments at HERA. In particular, substantial samples of reconstructed $D^{*}$ hadrons have been obtained, and a semi-lepton decay mode analysis is underway[6]. Considerably more data is anticipated in the next few years.

Interest in the production mechanism is twofold. First, one is concerned with the leading twist-two term in the operator product expansion which incorporates the factorization theorem for hard scattering. Second, there is interest in studies of a higher twist charm component to the proton.

\footnotetext{
* Invited lecture presented at Ringberg Workshop: New Results from HERA, 30 May - 4 June 1999, Schloß Ringberg, Rottach-Egern, Germany, to appear in proceedings thereof
}

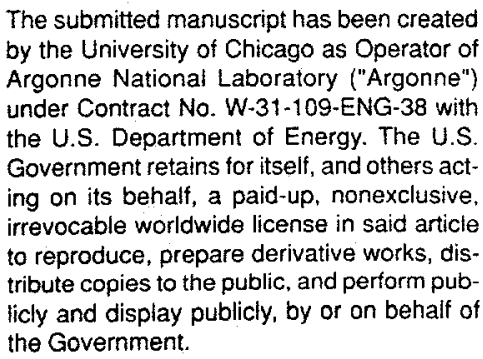




\section{DISCLAIMER}

This report was prepared as an account of work sponsored by an agency of the United States Government. Neither the United States Government nor any agency thereof, nor any of their employees, make any warranty, express or implied, or assumes any legal liability or responsibility for the accuracy, completeness, or usefuiness of any information, apparatus, product, or process disclosed, or represents that its use would not infringe privately owned rights. Reference herein to any specific commercial product, process, or service by trade name, trademark, manufacturer, or otherwise does not necessarily constitute or imply its endorsement, recommendation, or favoring by the United States Government or any agency thereof. The views and opinions of authors expressed herein do not necessarily state or reflect those of the United States Government or any agency thereof. 


\section{DISCLAIMER}

Portions of this document may be illegible in electronic image products. Images are produced from the best available original document. 
When considering the leading twist-two term the main issue is whether and when to treat the charm as a parton. Near threshold it is well established that charm is produced through photon-gluon fusion. On the other hand, very far above threshold the charm should be resummed into an effective parton distribution. How one interpolates from one region to another is described by a variable flavor number scheme, several of which have been proposed recently [7-9]. One must look at sufficiently inclusive observables in order to build up the logarithms that are to be summed, so predictive power for some observables is lost. In other words, for differential quantities, the fixed flavor number scheme of photon-gluon fusion provides the most appropriate formalism [10].

The idea of a higher twist charm component to the proton was introduced by Brodsky et al. [11]. In this scenario the intrinsic charm quark Fock component in the proton wave function, $|u u d c \bar{c}\rangle$, is generated by virtual interactions where gluons couple to two or more valence quarks. The probability for $c \bar{c}$ fluctuations to exist in a light hadron scales as $\mathcal{O}\left(1 / m_{c}^{2}\right)$ relative to the twist-two component, and is therefore higher twist. The EMC data [2] marginally support this idea. A full re-analysis of the EMC data has been carried out including both leading twist and intrinsic components at NLO including mass and threshold effects [12]. The result is that one data point contributes to a $0.8 \pm 0.6 \%$ normalization of the intrinsic component relative to leading twist.

Fragmentation is the most contentious topic at present. The production and subsequent hadronization of charmed quarks in DIS is not as clean as the much studied case of production in $e^{+} e^{-}$annihilation. In particular, in DIS there are proton beam remnants around which necessarily talk to the charm quark as it hadronizes. If events too close to the beam direction are selected, one can expect deviations from models which do not account for this effect. Presently it is hoped that detailed experimental studies in the Breit frame, wherein one hemisphere resembles $e^{+} e^{-}$, will provide additional information on the fragmentation process.

Because the production method is dominated by the leading twist photongluon fusion near threshold, it is possible to extract a gluon parton distribution function (PDF) and compare with existing PDFs, which derive their gluon information from other sources, such as direct photon production or structure function scaling violation measurements. This has been done by both $\mathrm{H} 1$ and ZEUS collaborations. The results are completely consistent with comparable PDF sets in the same $x$ and $Q^{2}$ range. While unlikely to ever replace structure function scaling violation measurements, charm production does serve as a nice consistency check on the gluon PDF.

One may also wish to take the open charm measurement and extrapolate the cross section over the full phase space and then extract the charm's contribution to the proton structure function. Historically the structure functions have been useful as input into global analyses and a testing ground for the variable flavor number schemes mentioned above. Care must be taken so that conclusions drawn are not artifacts of the extrapolation procedure (model). 


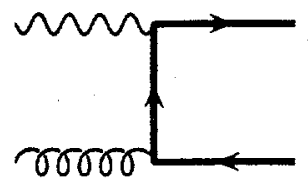

(b)

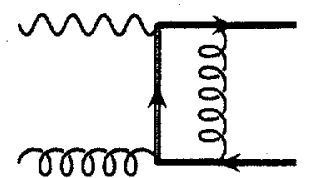

(b)

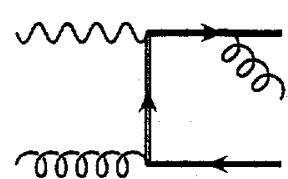

(c)

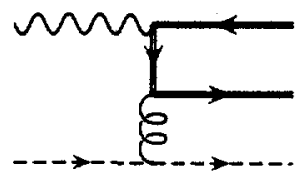

(d)

Fig. 1. Typical Feynman diagrams contributing to the amplitude for neutral current charm production. (a) An order $e g_{s}$ Born diagram. (b) An order $e g_{s}^{3}$ virtual diagram. (c) An order $e g_{s}^{2}$ gluon-bremsstrahlung diagram. (d) An order $e g_{s}^{2}$ light quark initiated diagram

\section{Next-to-leading Order Calculations}

The reaction under consideration is charm quark production via neutral-current electron-proton scattering, $e^{-}(l)+P(p) \rightarrow e^{-\left(l^{\prime}\right)}+c\left(p_{1}\right)+X$. When the momentum transfer squared $Q^{2}=-q^{2}>0\left(q=l-l^{\prime}\right)$ is not too large $Q^{2} \ll M_{Z}^{2}$, the contribution from $Z$ boson exchange is suppressed and the process is dominated by virtual-photon exchange. After an azimuthal integration, the cross section may be written in terms of structure functions $F_{2}^{c}\left(x, Q^{2}, m_{c}\right)$ and $F_{L}^{c}\left(x, Q^{2}, m_{c}\right)$ as follows:

$$
\frac{d^{2} \sigma}{d y d Q^{2}}=\frac{2 \pi \alpha^{2}}{y Q^{4}}\left\{\left[1+(1-y)^{2}\right] F_{2}^{c}\left(x, Q^{2}, m_{c}\right)-y^{2} F_{L}^{c}\left(x, Q^{2}, m_{c}\right)\right\}
$$

where $x=Q^{2} / 2 p \cdot q$ and $y=p \cdot q / p \cdot l$ are the usual Bjorken scaling variables, $\alpha$ is the electromagnetic coupling, and $m_{c}$ is the charm mass. The scaling variables are related to the square of the center of momentum energy of the electronproton system $S=(l+p)^{2}$ via $x y S=Q^{2}$. The total cross section is given by [13]

$$
\sigma=\int_{4 m_{c}^{2} / S}^{1} d y \int_{m_{e}^{2} y^{2} /(1-y)}^{y S-4 m_{c}^{2}} d Q^{2}\left(\frac{d^{2} \sigma}{d y d Q^{2}}\right)
$$

where $m_{e}$ is the electron mass.

Typical Feynman diagrams for this process are shown in Fig. 1. The interference of the $\mathcal{O}\left(e g_{s}\right)$ Born diagrams with the $\mathcal{O}\left(e g_{s}^{3}\right)$ one-loop virtual diagrams produces a result that is $\mathcal{O}\left(\alpha \alpha_{s}^{2}\right)$. The ultraviolet divergences are removed by renormalization in the Collins-Wilczek-Zee scheme [14]. The result is added to the square of the gluon-bremsstrahlung diagrams which is also $\mathcal{O}\left(\alpha \alpha_{s}^{2}\right)$. Initial state collinear singularities are mass factorized to obtain a finite NLO result. For the light quark initiated subprocess there are no virtual contributions at $\mathcal{O}\left(\alpha \alpha_{s}^{2}\right)$. One only encounters initial state collinear singularities which are again removed using factorization. The full NLO corrections were first calculated in $[15]$ and may be written in the form 


$$
\begin{aligned}
F_{k}^{c}\left(x, Q^{2}, m_{c}\right)= & \frac{Q^{2} \alpha_{s}\left(\mu^{2}\right)}{4 \pi^{2} m_{c}^{2}} \int_{\xi_{\min }}^{1} \frac{d \xi}{\xi}\left\{e_{c}^{2} f_{g / P}\left(\xi, \mu^{2}\right)\right. \\
& \times\left[c_{k, g}^{(0)}+4 \pi \alpha_{s}\left(\mu^{2}\right)\left(c_{k, g}^{(1)}+\bar{c}_{k, g}^{(1)} \ln \frac{\mu^{2}}{m_{c}^{2}}\right)\right] \\
+ & 4 \pi \alpha_{s}\left(\mu^{2}\right) \sum_{i=q, \bar{q}} f_{i / P}\left(\xi, \mu^{2}\right) \\
& \left.\times\left[e_{c}^{2}\left(c_{k, i}^{(1)}+\bar{c}_{k, i}^{(1)} \ln \frac{\mu^{2}}{m_{c}^{2}}\right)+e_{i}^{2} d_{k, i}^{(1)}+e_{i} e_{c} o_{k, i}^{(1)}\right]\right\}
\end{aligned}
$$

with $k=2, L$. The lower limit on the integration over the parton momentum fraction $\xi$ is $\xi_{\min }=x\left(4 m_{c}^{2}+Q^{2}\right) / Q^{2}$. The parton momentum distributions in the proton are denoted by $f_{i / P}\left(\xi, \mu^{2}\right)$. The sum is taken over the light quarks, $q=u, d, s$. The mass factorization scale $\mu_{f}$ has been set equal to the renormalization scale $\mu_{r}$ and is denoted by $\mu$. All charges are in units of $e . c_{k, i}^{(0)}, c_{k, i}^{(1)}$, $\bar{c}_{k, i}^{(1)},(i=g, q, \bar{q})$, and $d_{k, i}^{(1)}, o_{k, i}^{(1)},(i=q, \bar{q})$ are scale independent parton coeffcient functions, and are distinguished by their origin. The $c$-coefficient functions originate from processes involving the virtual photon-heavy quark coupling, the $d$-coefficient functions arise from processes involving the virtual photon-light quark coupling, and the o-coefficient functions are from the interference between these processes.

In addition to describing the structure functions, the coefficient functions of [15] may also be used to compute the single-inclusive distributions $d F_{k} / d p_{t}$ and $d F_{k} / d y[16]$, where $p_{t}$ and $y$ are the transverse momentum and rapidity, respectively, of the heavy quark in the virtual photon-proton center of momentum system.

Unfortunately, the analytic expressions of the coefficient functions are too long to be published in journal form. They are however available as computer code, but, for the same reason, the code tends to be slow. Initially this was seen as an impediment to including them in a global fitting program. Then in [17], with the threshold and asymptotic behavior of the coefficient functions removed, it was possible to numerically tabulate grids, with a fast interpolation routine, so that speedy computation of Eq. (1) - (3) became possible. This process was further refined in [18].

The coefficient functions in the large momentum transfer limit are a necessary ingredient for constructing the variable flavor number schemes mentioned in the introduction. Exact analytic formulae for the $d_{k, i}^{(1)}$ together with analytic formulae for the coefficient functions $c_{k, i}^{(0)}, c_{k, i}^{(1)}, \bar{c}_{k, i}^{(1)},(i=g, q, \bar{q})$, and $d_{k, i}^{(1)},(i=q, \bar{q})$ in the limit $Q^{2} \gg m^{2}$ can be found in [19].

The coefficient functions have also been calculated in a fully differential form [20]. These in turn can be used with Eq. (3) to construct pair-inclusive distributions such as $d F_{k} / d M_{c \bar{c}}$ [21] where $M_{c \bar{c}}$ is the invariant mass of the produced charm-anticharm system. 
The resulting differential structure functions and Eq. (1) have further been used to construct a NLO monte carlo style program, HVQDIS $[22,23]$. The basic components (in terms of virtual-photon-proton scattering) are the 2 to 2 body squared matrix elements through one-loop order and tree level 2 to 3 body squared matrix elements, for both photon-gluon and photon-light-quark initiated subprocesses, as shown in Fig. 1. It is therefore possible to study fully-, single-, and semi-inclusive production at NLO, and three body final states at leading order. The goal of this NLO calculation is to organize the soft and collinear singularity cancellations without loss of information in terms of observables that can be predicted.

The subtraction method provides a mechanism for this cancellation. It allows one to isolate the soft and collinear singularities of the 2 to 3 body processes within the framework of dimensional regularization without calculating all the phase space integrals in a space-time dimension $n \neq 4$. Expressions for the threebody squared matrix elements in the limit where an emitted gluon is soft appear in a factorized form where poles $\epsilon=2-n / 2$ multiply leading order squared matrix elements. These soft singularities cancel upon addition of the interference of the leading order diagrams with the renormalized one-loop virtual diagrams. The remaining singularities are initial state collinear in origin. The three-body squared matrix elements appear in a factorized form, where poles in $\epsilon$ multiply splitting functions convolved with leading order squared matrix elements. These collinear singularities are removed through mass factorization.

The result of this calculation is an expression that is finite in four-dimensional space time. One can compute all phase space integrations using standard monte carlo integration techniques. The final result is a program which returns parton kinematic configurations and their corresponding weights, accurate to $\mathcal{O}\left(\alpha \alpha_{s}^{2}\right)$. The user is free to histogram any set of infrared-safe observables and apply cuts, all in a single histogramming subroutine. Additionally, one may study heavy hadrons using the Peterson et al. model [24]. Detailed physics results from this program and a description of the necessary cross checks the program satisfies are given in [23]. See also [25] for recent improvements.

\section{Results and Current Issues}

Charmed meson differential cross sections are measured experimentally $[1,2,4,5]$ within some detector acceptance region, and the corresponding theory predictions can be made using HVQDIS. As we saw above, the cross section is an integral over the structure functions. Therefore, the two share many of the same properties. Before discussing the cross sections, the more salient features of the NLO structure functions will be reviewed. The interested reader can find additional details in the original paper [15] and, more so, in the recent phenomenological analyses $[18,26-28]$.

For moderate $Q^{2} \sim 10 \mathrm{GeV}^{2}$ one finds that the charm quark contribution at small $x \sim 10^{-4}$ is approximately $25 \%$ of the total structure function (defined as light parton plus heavy quark contributions). In contrast, the contribution from 

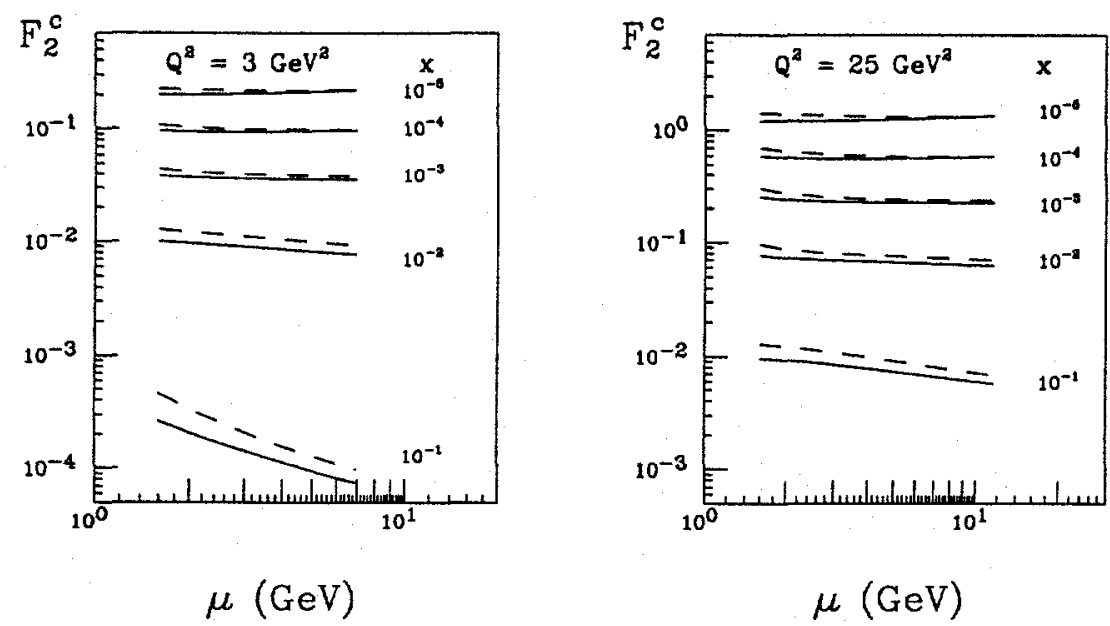

Fig. 2. The renormalization/factorization scale dependence of the structure function $F_{2}^{c}\left(x, Q^{2}, m_{c}\right)$ for $Q^{2}=3 \mathrm{GeV}^{2}$ (left) and $Q^{2}=25 \mathrm{GeV}^{2}$ (right) for various $x$ values. The results for GRV94 (solid lines) and CTEQ4F3 (dashed lines) parton distribution sets are shown

bottom quarks is only a few percent due to charge and phase space suppression. The structure functions show a marked rise at small $x$ due primarily to the rapidly rising gluon distribution: the gluon initiated contributions comprise most of the structure function. The light quark initiated processes give only a few percent contribution at small $x$. The scale dependence of the structure functions is very small in the HERA $x$ and $Q^{2}$ regions. This is demonstrated in Fig. 2 for various $x$ and $Q^{2}$ values. The largest variation comes from our imprecise knowledge of the charm quark mass. For example, a $\pm 10 \%$ variation of the charm mass about the central value of $1.5 \mathrm{GeV}$ gives a $\pm 20 \%$ variation in the structure function for small $x$ and moderate $Q^{2}$.

At moderate $Q^{2}$, and $x$ values larger than 0.01 , the charm structure function is increasingly dominated by partonic processes near the charm quark pairproduction threshold. The large size of the gluon density for small momentum fractions gives relatively large weight to such processes [27]. Although the QCD corrections at presently accessible $x$ values are moderate (about $30-40 \%$ ), with an increasing amount of data to be gathered at higher $x$, it is worthwhile to have a closer look at such near-threshold subprocesses. In this kinematic region, the QCD corrections are dominated by large Sudakov double logarithms. Recently [29], these Sudakov logarithms have been resummed to all orders of perturbation theory, to next-to-leading logarithmic accuracy, and, moreover, in single-particle inclusive kinematics [30]. Let us recall the main results. First, the quality of the approximation for the next-to-leading logarithmic threshold resummation was found to be clearly superior to leading logarithmic one. Furthermore, the resum- 

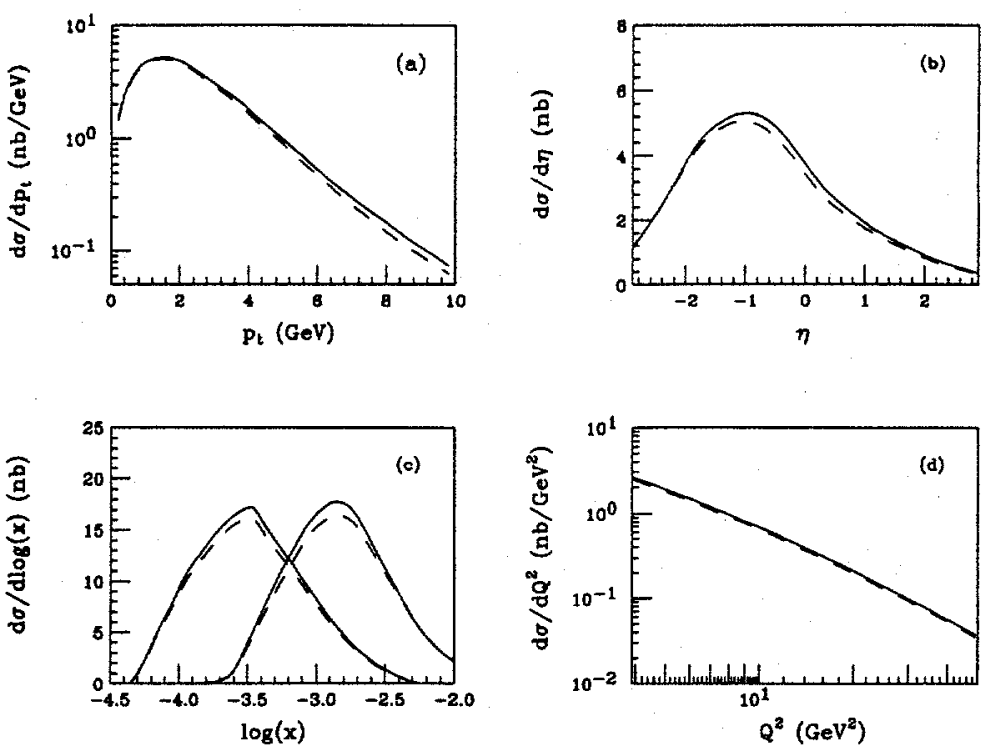

Fig. 3. Next-to-leading order differential cross sections for charm production at $\sqrt{S}=$ $301 \mathrm{GeV}$ at HERA using the GRV94 (dashed lines) and CTEQ4F3 (solid lines) parton distribution sets. (a) Transverse momentum. (b) Pseudo-rapidity. (c) Bjorken $x$ (left set) and parton momentum fraction $\xi$ (right set). (d) Photon virtuality $Q^{2}$

mation provided next-to-next-to-leading order estimates [29], which were found to be sizable for $x \geq 0.05$.

Let us now examine some of the properties of the charm quark cross section. Results are presented in the HERA lab frame with positive rapidity in the proton direction. The proton and electron beam energies are taken to be $820 \mathrm{GeV}$ and $27.6 \mathrm{GeV}$, respectively. Results are for the kinematic range $3<Q^{2}<50 \mathrm{GeV}^{2}$ and $0.1<y<0.7$. The CTEQ4F3 [31] and GRV94 HO [32] proton-parton distribution sets are used. The renormalization and factorization scales have been set equal to $\mu$.

Fig. 3 shows the NLO cross sections differential in transverse momentum $p_{t}$, pseudo-rapidity $\eta$, Bjorken $x$, and momentum transfer $Q^{2}$ for charm quark production using the GRV94 (dashed) and CTEQ4F3 (solid) parton distribution sets at $\mu=\sqrt{Q^{2}+4 m_{c}^{2}}$ with $m_{c}=1.5 \mathrm{GeV}$. From Eq. (3) the parton distributions are probed at a momentum fraction $\xi$ which is typically one order of magnitude larger the $x$. This is illustrated in Fig. 3c where a plot of $d \sigma / d \log (\xi)$ vs. $\log (\xi)$ (right set of curves) is superimposed on the plot of $d \sigma / d \log (x)$ vs. $\log (x)$ (left set of curves). The difference between the curves is approximately $10 \%$ at $\xi=10^{-2.7}$.

The scale dependence of the NLO differential cross sections is shown in Fig. 4. The curves were made using the CTEQ4F3 parton distribution set at $\mu=2 m_{c}$ (solid) and $\mu=2 \sqrt{Q^{2}+4 m_{c}^{2}}$ (dashed) with $m_{c}=1.5 \mathrm{GeV}$. The curves show 

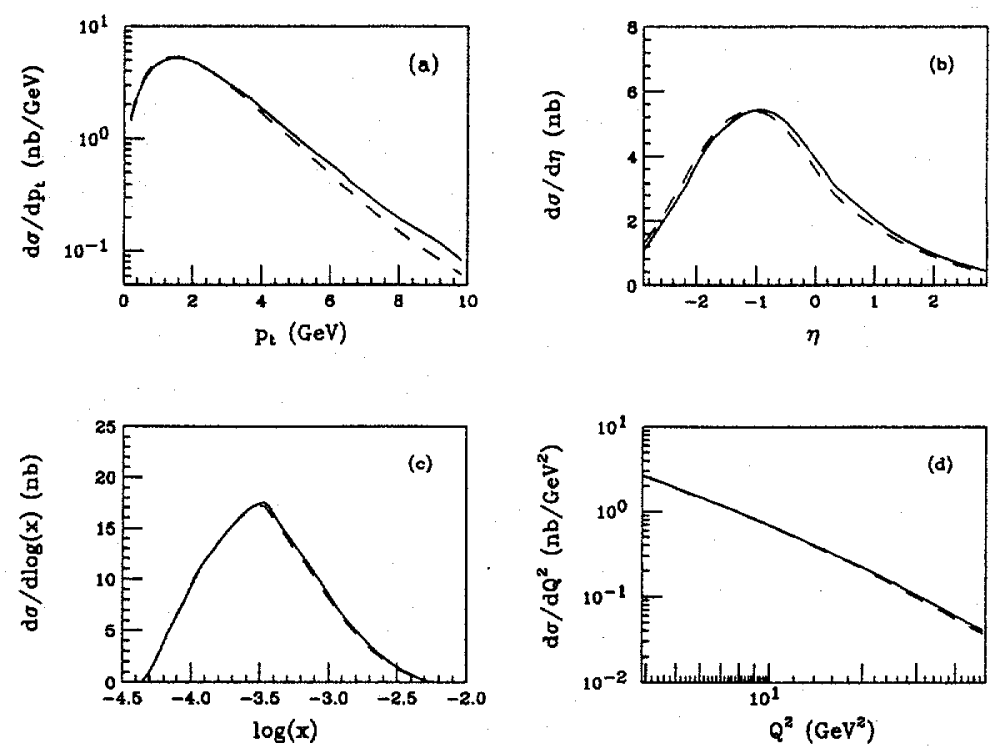

Fig. 4. Same set of distributions as Fig. 3, but this time showing the variation with respect to renormalization/factorization scale, $\mu=2 m_{c}$ (solid lines) and $\mu=$ $2 \sqrt{Q^{2}+4 m_{c}^{2}}$ (dashed lines)

very little scale dependence. This can be anticipated from the results shown in Fig. 2 and the distribution in Bjorken $x$ shown in Fig. 4c. The latter shows the cross section is dominated by $x \sim 10^{-3.5}=3.2 \times 10^{-4}$ while the former shows that, independent of $Q^{2}$, the structure function is very flat in this particular $x$ region. Therefore, the cross section tends to be fairly insensitive to the choice of scale. Other kinematic regions show increased scale dependence.

The largest uncertainty in the structure function calculation is due to the charm quark mass. The same is true for the cross section as shown in Fig. 5. The NLO differential cross sections for charm quark production using the CTEQ4F3 parton distribution set at $\mu=\sqrt{Q^{2}+4 m_{c}^{2}}$ with $m_{c}=1.35 \mathrm{GeV}$ (solid) and $m_{c}=1.65 \mathrm{GeV}$ (dash) are shown. Mass effects are smaller at the larger transverse mass because they are suppressed by powers of $m_{c} / p_{t}$ in the matrix elements. However, if the range is extended much further, large logarithms of the form $\ln \left(p_{t}^{2} / m_{c}^{2}\right)$ appear in the cross section and should be resummed.

Before closing, we compare the NLO calculations described above with the most recent HERA data from H1 [4] and ZEUS [5] collaborations. The measurements make use of a tagging technique wherein the $D^{*}$ meson kinematics are reconstructed using the tight constrains of the decay $D^{*+} \rightarrow D^{0} \pi_{\text {slow }}^{+} \rightarrow$ $\left(K^{-} \pi^{+}\right) \pi_{\text {slow }}^{+}$. In order to make the comparison, the theory prediction for the charm production cross sections must be converted to those of charmed meson production. This is done using a simple non-evolving Peterson et al. model [24] which depends on one parameter $\epsilon$ which is taken from $e^{+} e^{-}$data. The overall 

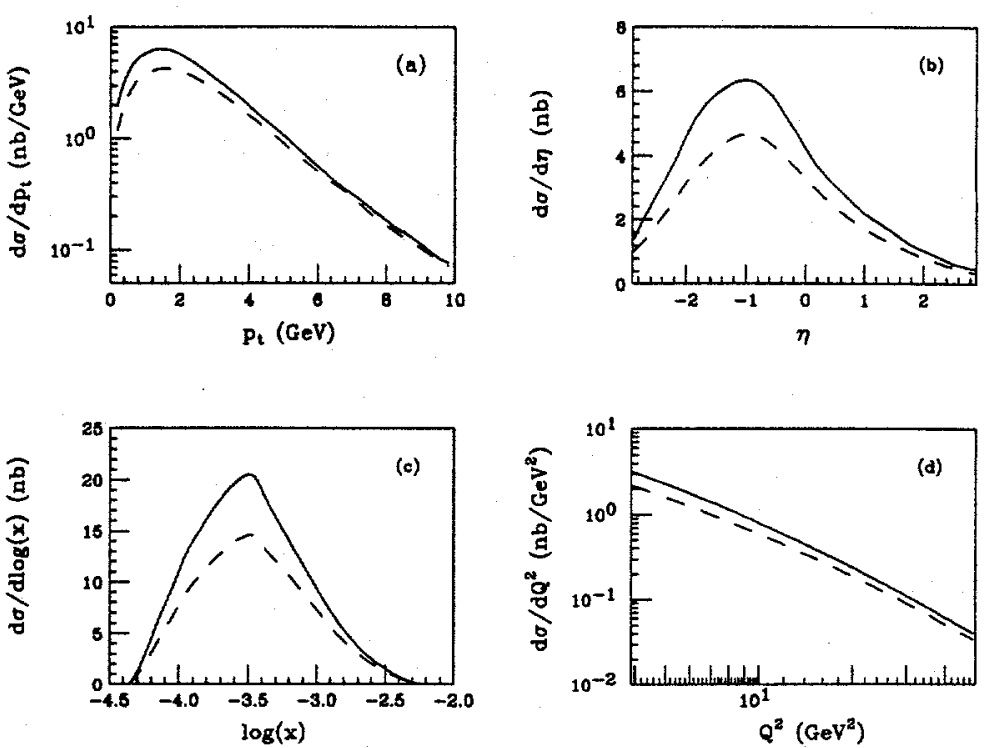

Fig. 5. Same set of distributions as Fig. 3, but this time showing the variation with respect to charm quark mass, $m_{c}=1.35 \mathrm{GeV}$ (solid lines) and $m_{c}=1.65 \mathrm{GeV}$ (dashed lines )

cross section normalization is set by the hadronization fraction $f\left(c \rightarrow D^{*}\right)$ again taken from $e^{+} e^{-}$data. The four-vector for the $D^{*}$ is constructed from that of the charmed quark by smearing the the charm three-vector in the lab frame. The energy component is then fixed such that the four-vector has the physical $D^{*}$ mass, $2.01 \mathrm{GeV}$.

Shown in Fig. 6 are $D^{*}$ meson cross sections measured by the $\mathrm{H} 1$ collaboration [4] differential in transverse momentum $p_{\perp}$, pseudo-rapidity $\eta$, reconstructed parton momentum fraction $x_{g}^{\text {obs }}$, and momentum transfer $Q^{2}$ compared to the NLO calculation described in the Sec. 2 plus the Peterson et al. fragmentation model. The shaded band corresponds to varying the charm quark mass of $\mathbf{1 . 5}$ $\mathrm{GeV}$ by $\pm 0.2 \mathrm{GeV}$. Overall the agreement is good, except for the pseudo-rapidity plot in which the theory under (over) estimates the data in the forward (backward) region.

The ZEUS collaboration [5] has also measured $D^{*}$ meson cross sections differential in momentum transfer $Q^{2}$, Bjorken $x$, hadronic energy $W$, transverse momentum $p_{t}$, pseudo-rapidity $\eta$, and $D^{*}$ momentum fraction $x\left(D^{*}\right)=$ $2\left|\boldsymbol{p}_{\gamma \mathrm{Pcms}}\right| / W$ which are compared with theory in Fig. 7 . The boundaries of the bands correspond to varying the charm quark mass of $1.4 \mathrm{GeV}$ by $\pm 0.1 \mathrm{GeV}$. Again, the overall agreement is good, but the theory underestimates the data in the forward region and overestimates it in the backward region. Additionally, the $D^{*}$ momentum fraction data, which is particularly sensitive to the charm 

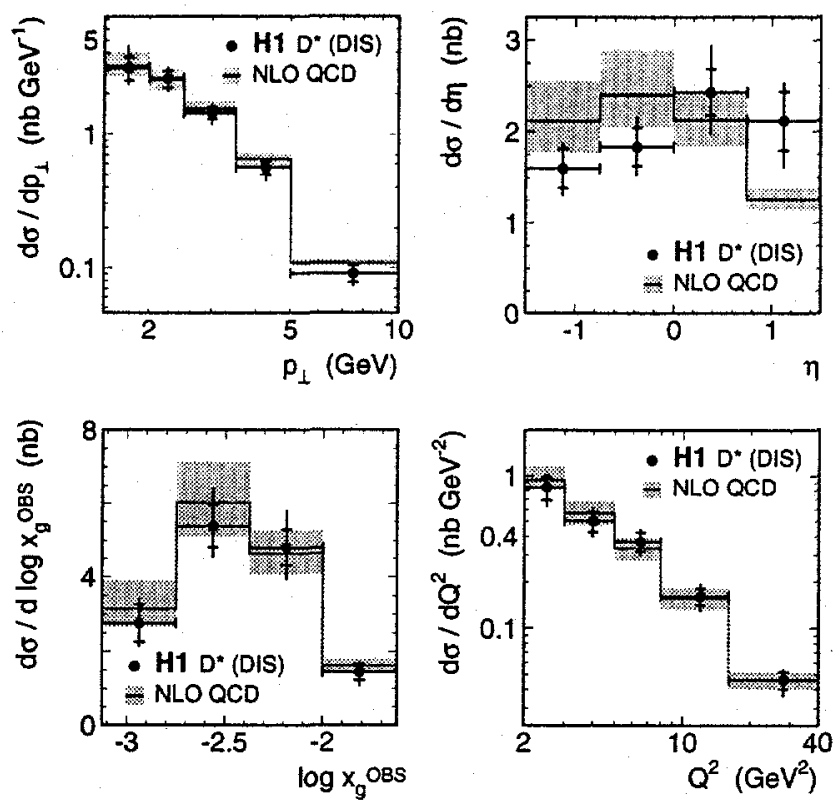

Fig. 6. Various differential cross sections for $D^{*}$ meson production at HERA as measured by the $\mathrm{H} 1$ collaboration [4] compared to the next-to-leading order calculation described in the Sec. 2 plus a Peterson et al. fragmentation model. The shaded band corresponds to varying the charm quark mass from 1.3 to $1.7 \mathrm{GeV}$

hadronization process, is poorly described. Similar effects are seen in the $D^{*}$ photoproduction data at HERA.

Variations of the parton distribution set, renormalization/factorization scale, charm mass, or fragmentation parameter $\epsilon$ are unable to account for the differences between data and theory. It also appears unlikely that an evolving fragmentation function would help; and the momentum transfers are large enough that any photon structure is surely negligible.

One explanation[33] proposed for the photoproduction data appears to work for the DIS data as well. Qualitatively, one is invited to think of a color string connecting the hadronizing charm quark and the proton remnant which pulls (drags) the charmed meson to the forward region. This is made quantitative in the Lund String model modified for heavy flavor production [34], as implemented in Pythia [35]. The shaded band in Fig. 7 shows what happens when the Peterson et al. model is replaced by an effective fragmentation model extracted from the Pythia based monte carlo RAPGAP[36]. The agreement is much improved.

Another way to improve the agreement between data and theory is to simply raise the minimum $p_{t}$ of the events that are selected. Data from a slightly different decay chain, but higher minimum $p_{t}$ cut are shown as open triangles. Here the Peterson and RAPGAP improved NLO predictions give essentially the same 


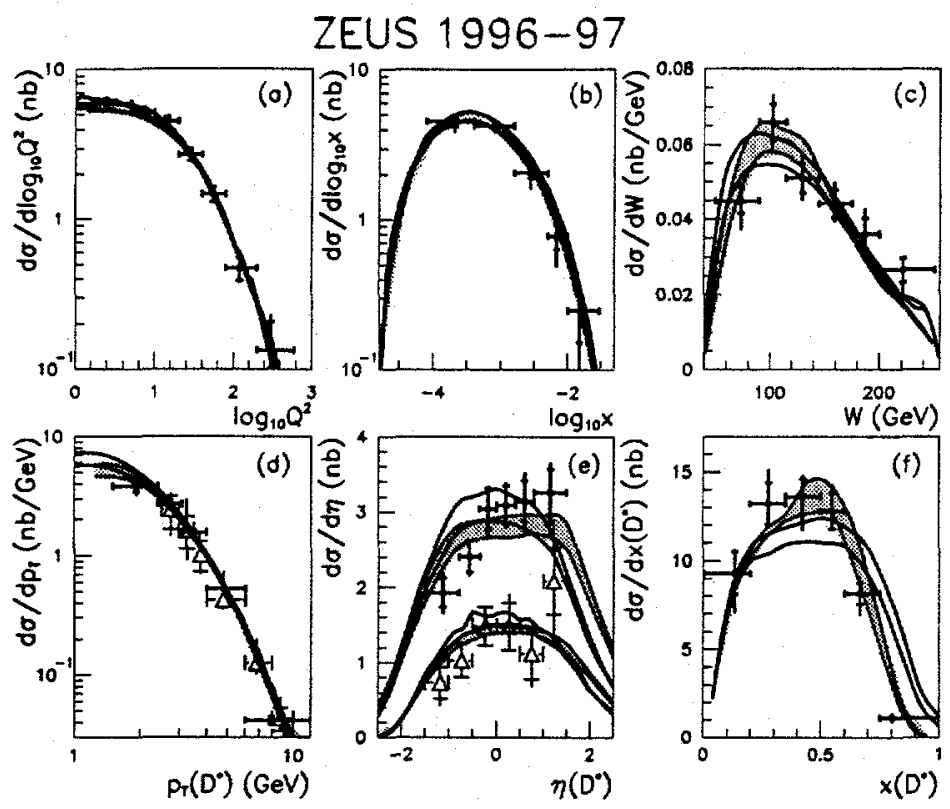

Fig. 7. Various differential cross sections for $D^{*}$ meson production at HERA as measured by the ZEUS collaboration [5] compared to the next-to-leading order calculation described in the Sec. 2 plus a Peterson et al. fragmentation model. For the shaded band the Peterson et al. model was replaced by an effective fragmentation model extracted from RAPGAP which includes a drag effect between the proton remnant and the hadronizing charm. The bands result from varying the charm quark mass from 1.3 to $1.5 \mathrm{GeV}$

results, as expected. However, a seemingly large fluctuation in the forward most data bin somewhat clouds this expectation.

A number of additional studies have been done. For example, H1 [4], using the above cross sections, has extracted a NLO gluon PDF which agrees well with their own gluon PDF, obtained indirectly through structure function scaling violations, and that of CTEQ4F3. In a different approach, ZEUS [5], has extrapolated over the full phase space and extracted the structure function $F_{2}^{\text {charm. }}$

In closing, the next-to-leading order calculations described herein have been very successful in describing charm production at HERA. A variety of different observables have been studied, and the gluon parton distribution function and the charm contribution to proton structure function have been extracted. The weakest stage of the calculation is, not surprisingly, modeling the hadronization of the produced charm to the observed charmed meson, especially at low trans- 
verse momentum. One could take this as an opportunity to study hadronization in the presence of beam remnants.

Acknowledgments. I thank the organizers for the invitation and Z. Sullivan for comments on the text. The work presented herein is the result of collaborations and discussions with J. Smith and E. Laenen, and was supported in part by the U.S. Department of Energy, High Energy Physics Division, Contract No. W-31-109-Eng-38.

\section{References}

1. BFP Collaboration, G. D. Gollins et al.: Phys. Rev. D24, 559 (1981)

2. EMC Collaboration, J. J. Aubert et al.: Nucl. Phys. B213, 31 (1983)

3. M. Strovink: 'Review of multimuon production by muons'. In: Proceedings of the 1981 International Symposium on Lepton and Photon Interactions at High Energy, Bonn, 1981, edited by W. Pfeil (Bonn Univ., Phys. Inst. 1981) pp. 594-622

4. H1 Collaboration, C. Adloff et al.: Z. Phys. C72, 593 (1996); Nucl. Phys. B545, 21 (1999)

5. ZEUS Collaboration, J. Breitweg et al.: Phys. Lett. B407, 402 (1997); DESY 99-101, hep-ex/9908012

6. Measurement of Charm Production in Deep Inelastic Scattering, W. Verkerke, $\mathrm{Ph} . D$. Thesis, University of Amsterdam, 1998.

7. M. Aivazis, J.C. Collins, F.I. Olness, and W.K. Tung: Phys. Rev. D50, 3102 (1994)

8. M. Buza, Y. Matiounine, J. Smith and W.L. van Neerven: Phys. Lett. B411, 211 (1997); Eur. Phys. J. C1, 301 (1998)

9. R. S. Thorne, R. G. Roberts: Phys. Rev. D57, 6871 (1998)

10. C. R. Schmidt: 'QCD phenomenology of charm production at HERA'. In: Proceedings of the 5th International Workshop on Deep Inelastic Scattering and QCD, Chicago, IL, 1997, edited by J. Repond and D. Krakauer (American Institute of Physics, Woodbury 1997) pp. 381-385

11. S. J. Brodsky, P. Hoyer, C. Peterson, and N. Sakai: Phys. Lett. B93, 451 (1980); S. J. Brodsky, C. Peterson, and N. Sakai: Phys. Rev. D23, 2745 (1981)

12. B. W. Harris, J. Smith, and R. Vogt: Nucl. Phys. B461, 181 (1996)

13. G.A. Schuler: Nucl. Phys. B299, 21 (1988)

14. J.C. Collins, F. Wilczek and A. Zee: Phys. Rev. D18, 42 (1978)

15. E. Laenen, S. Riemersma, J. Smith, and W.L. van Neerven: Nucl. Phys. B392, 162 (1993)

16. E. Laenen, S. Riemersma, J. Smith, and W.L. van Neerven: Nucl. Phys. B392, 229 (1993)

17. S. Riemersma, J. Smith, and W.L. van Neerven: Phys. Lett. B347, 143 (1995)

18. K. Daum, S. Riemersma, B. W. Harris, E. Laenen, and J. Smith: 'The heavyflavour contribution to proton structure'. In: Proceedings of the Workshop 1995/96 on Future Physics at HERA, Hamburg, 1996, edited by G. Ingelman et al. (Deutches Elektronen-Synchrotron, Hamburg 1996), p. 89.

19. M. Buza, Y. Matiounine, J. Smith and W.L. van Neerven: Nucl. Phys. B472, 611 (1996)

20. B. W. Harris and J. Smith: Nucl. Phys. B452 109 (1995)

21. B. W. Harris and J. Smith: Phys. Lett. B353, 535 (1995) 
22. B. W. Harris: 'Electroproduction of heavy quarks at NLO'. In: Minneapolis Meeting of the Division of Particles and Fields of the American Physical Society, Minneapolis, 1996, edited by J.K. Nelson and K. Heller (World Scientific, Singapore 1996) pp. 1019-1021

23. B. W. Harris and J. Smith: Phys. Rev. D57, 2806 (1998)

24. C. Peterson, D. Schlatter, I. Schmitt, and P.M. Zerwas: Phys. Rev. D27, 105 (1983)

25. B. W. Harris, E. Laenen, S. Moch, and J. Smith: 'Heavy quark production in deep-inelastic scattering at HERA'. In: Proceedings of Workshop on Monte Carlo Generators for HERA Physics, Hamburg, Germany, 1998, edited by G. Grindhammer, G. Ingelman, H. Jung, and T. Doyle (DESY, Hamburg 1998)

26. M. Glück, E. Reya, and M. Stratmann: Nucl. Phys. B422, 37 (1994)

27. A. Vogt, in International Workshop on Deep Inelastic Scattering and Related Phenomena (DIS 96), Rome, 1996, edited by G. D'Agostini and A. Nigro (World Scientific, Singapore 1997), p. 254.

28. E. Laenen, M. Buza, B. W. Harris, Y. Matiounine, R. Migneron, S. Riemersma, J. Smith, and W. L. van Neerven: 'Deep-inelastic production of heavy quarks'. In: Proceedings of the Workshop 1995/96 on Future Physics at HERA, Hamburg, 1996, edited by G. Ingelman et al. (Deutches Elektronen-Synchrotron, Hamburg 1996), p. 393.

29. E. Laenen and S. Moch: Phys. Rev. D59, 034027 (1999)

30. E. Laenen, G. Oderda and G. Sterman, Phys. Lett. B438, 173 (1998)

31. H. L. Lai and W. K. Tung: Z. Phys. C74, 463 (1997)

32. M. Glück, E. Reya and A. Vogt: Z. Phys. C67, 433 (1995)

33. E. Norrbin and T. Sjöstrand: 'Drag effects in charm photoproduction'. In: Proceedings of Workshop on Monte Carlo Generators for HERA Physics, Hamburg, Germany, 1998, edited by G. Grindhammer, G. Ingelman, H. Jung, and T. Doyle (DESY, Hamburg 1998)

34. M. G. Bowler, Z. Phys. C11, 169 (1981)

35. T. Sjöstrand, Comp. Phys. Comm. 82, 74 (1994)

36. H. Jung: Comp. Phys. Comm. 86, 147 (1995) 